\title{
Magnetic anomalies possibly linked to local low seismicity
}

\author{
F. Masci, P. Palangio, and M. Di Persio \\ Istituto Nazionale di Geofisica e Vulcanologia, L'Aquila, Italy \\ Received: 20 May 2009 - Accepted: 26 August 2009 - Published: 18 September 2009
}

\begin{abstract}
During the last twenty years a time-synchronized network of magnetometers has operated in Central Italy along the Apennine chain to monitor the magnetic field anomalies eventually related to the tectonic activity. At present time the network consists of five stations. In the past only few anomalies in the local geomagnetic field, possibly associated to earthquakes, has been observed, not least because the network area has shown a low-moderate seismic activity with the epicentres of the few events with $M l \geq 5$ located away from the network station. During 2007 two $M l \approx 4$ earthquakes occurred in proximity of two stations of the network. Here we report the magnetic anomalies in the geomagnetic field that could be related with these tectonic events. To better investigate these two events a study of ULF (ultra-low-frequency) emissions has been carried out on the geomagnetic field components $\mathrm{H}, \mathrm{D}$, and $\mathrm{Z}$ measured in L'Aquila Observatory during the period from January 2006 to December 2008. We want to stress that this paper refers to the period before the 2009 L'Aquila seismic sequence which main shock $(M l=5.8)$ of 6 April heavily damaged the medieval centre of the city and surroundings. At present time the analysis of the 2009 data is in progress.
\end{abstract}

\section{Introduction}

Seismic activity can be associated to the anomalous variation of a wide kind of parameters. Some people link the unnatural behaviour of animals to large damaging earthquakes (Tributsch, 1983), or point out the increase in social tension several years before damaging earthquakes (Molchanov, 2008). Anyway, many papers show the evident occurrence of magnetic, electric and electromagnetic phenomena prior, during or after a tectonic event (Stacey, 1964; Hayakawa and Fujinawa, 1994; Johnston and Parrot, 1998). The observation of these anomalies is quite difficult. Their amplitude depends

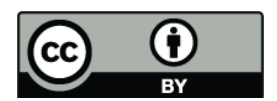

Correspondence to: F. Masci (masci@ingv.it) principally on the intensity of the seismic events, on the physical mechanisms involved, and on the distance of the observation point from the earthquake hypocenter. The principal mechanisms generating these electric and magnetic anomalies are: piezomagnetism, stress dependence of electrical resistivity, electrokinetic effects, charge generation processes and magnetohydrodynamic effects (Johnston, 1997). Usually only large earthquakes $(M l \geq 5)$ generate evident electromagnetic anomalies. Moreover, coseismic anomalies are larger than preseismic and postseismic events because the observed coseismic effects are due to the release of the accumulated crustal stress during the entire earthquake duration, whereas the preseismic signals are due to a small fraction of the accumulated energy release (Mueller and Johnston, 1998). Moreover, sometimes no precursory signals occurred before the earthquake, or precursory signals occurred with no corresponding coseismic signals. The Italian Istituto Nazionale di Geofisica e Vulcanologia (INGV) tectonomagnetic network was installed in Central Italy since the middle of 1989 to investigate the magnetic anomalies possibly related to earthquakes. The network is part of L'Aquila Geomagnetic Observatory and covers an area extending approximately in latitude range $\left[41^{\circ}-43^{\circ}\right] \mathrm{N}$ and longitude range $\left[12.5^{\circ}-15.0^{\circ}\right] \mathrm{E}$ (Masci et al., 2006, 2007, 2008). At present time, the network stations are: L'Aquila (AQU), Monte di Mezzo (MDM), Civitella Alfedena (CVT), Leonessa (LEO) and Duronia (DUR). Figure 1 shows the location of the stations in Central Italy, and in Table 1 their geographical coordinates are reported. In each station the total magnetic field intensity data are collected using proton precession magnetometers. The sampling interval of each station is set to 15 min except for AQU and DUR in which the sampling interval is of $1 \mathrm{~min}$. Moreover, AQU and DUR are equipped with triaxial fluxgate magnetometers with $1 \mathrm{~Hz}$ sampling rate. From the seismic point of view, Central Italy is an area with several active faults NW-SE bounded along the Apennine mountain chains. Bella et al. (1998) described anomalous acoustic, electric and magnetic signals related to the $M l=3.9$ Gran Sasso earthquake occurred on 25 August 1992. Plastino and Bella (2001) reported some anomalies in

Published by Copernicus Publications on behalf of the European Geosciences Union. 


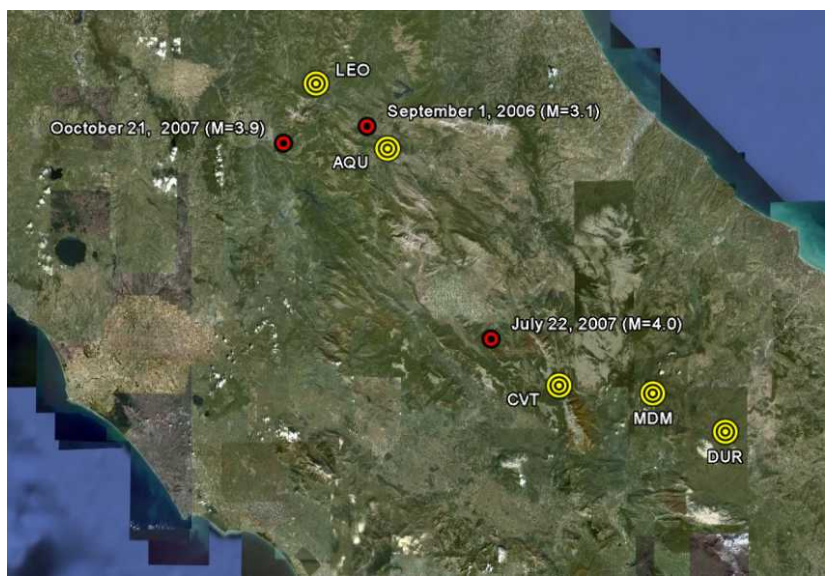

Fig. 1. Yellow-black points: location of the INGV tectonomagnetic network stations in Central Italy. Red-black points: epicentres of three tectonic events occurred in proximity of the stations. $M$ refers to the earthquakes local magnitude.

Table 1. Geographical coordinates of the INGV tectonomagnetic network stations.

\begin{tabular}{llll}
\hline AQU & $42^{\circ} 23^{\prime} \mathrm{N}$ & $13^{\circ} 19^{\prime} \mathrm{E}$ & $682 \mathrm{~m}$ a.s.l. \\
CVT & $41^{\circ} 47^{\prime} \mathrm{N}$ & $13^{\circ} 54^{\prime} \mathrm{E}$ & $1020 \mathrm{~m}$ a.s.l. \\
DUR & $41^{\circ} 39^{\prime} \mathrm{N}$ & $14^{\circ} 27^{\prime} \mathrm{E}$ & $910 \mathrm{~m}$ a.s.l. \\
LEO & $42^{\circ} 33^{\prime} \mathrm{N}$ & $13^{\circ} 04^{\prime} \mathrm{E}$ & $1320 \mathrm{~m}$ a.s.l. \\
MDM & $41^{\circ} 46^{\prime} \mathrm{N}$ & $14^{\circ} 13^{\prime} \mathrm{E}$ & $980 \mathrm{~m}$ a.s.l. \\
\hline
\end{tabular}

electrical conductivity, $\mathrm{PH}$ and radon content in Gran Sasso groundwater related to local seismic activity. In addition to that, several studies have shown in Central Italy the relationship between anomalous decrease in the VLF-LF radio signals and low (Biagi et al., 2007) or moderate (Biagi et al., 2004, 2008) seismic activity. From the geomagnetic point of view, Central Italy area is characterized by the presence of granulitic and titano-magnetite rocks and shows on average a low-medium crustal magnetization intensity (Molina et al., 1994). At the beginning of the 1990s an anomaly of few $\mathrm{nT}$ in the geomagnetic field, has been observed during two months by the INGV tectonomagnetic network (Mele et al., 1994). At the end of May 2003, Di Mauro et al. (2008) by means of wavelet analysis found a remarkable power enhancement in the dynamic spectra related to the MDM signal, few days before the earthquake $(M l=4.1)$ occurred on 1 June 2003 about $50 \mathrm{~km}$ at east of MDM station.

\section{$2 \quad 2007$ events}

Figure 2 shows the 2007 datasets of the network stations of AQU, MDM and CVT. The data of LEO station are not reported because of the large number of gaps due to technical

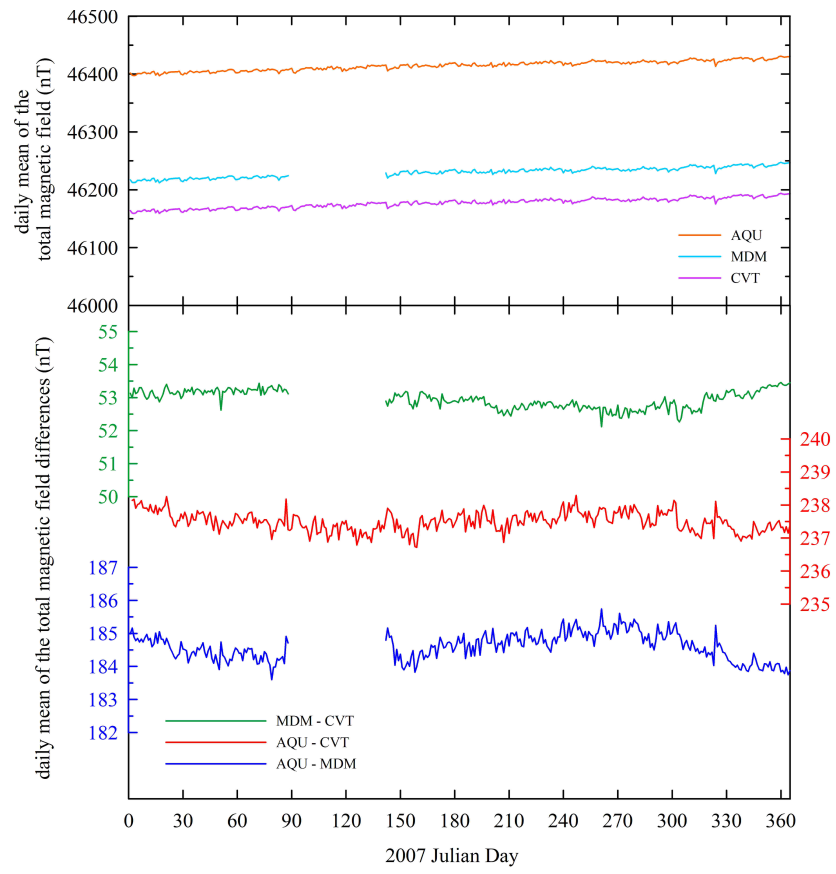

Fig. 2. 2007 data set of the network stations AQU, MDM and CVT. Top: daily means of the total magnetic field. Bottom: daily means of the total magnetic field differences for the couple of stations AQU-CVT, AQU-MDM, MDM-CVT. The colour of each plot corresponds to the colour of the vertical axis.

problems, while the DUR data are not shown because the station started its activity at the end of 2007. The top panel of Fig. 2 shows the 2007 datasets as daily means of the total magnetic field while the bottom panel shows the daily means of the differences of the synchronously sampled signals of AQU, CVT and MDM stations. The daily mean of the differentiated data is calculated to remove the diurnal variation. This simple differentiation of the total magnetic field data reduces ionospheric and magnetospheric disturbances, and removes the secular trend of the geomagnetic field. The remaining signal is due to the local variation in crustal magnetization and it is possibly related to tectonic activity as well. According with this simple procedure some authors found magnetic anomalies as offsets of the total magnetic field differences in correspondence of tectonic events. The amplitude of the offsets ranges from tenths to few nT, while their appearance time can be rapid or relatively slow. These offsets can persist for a long period (years), as in the case of $M l=5.9$ North Palm Spring earthquake of 8 July 1986, or are limited in time up to weeks or months (see the review papers of Muller and Johnston, 1998 and Johnston, 1997). In the later case the signal recovers to the level preceding the anomaly. Moreover, Johnston and Mueller (1987) reported an increase of the local geomagnetic field during a four months period prior to the earthquake. In the past centuries, several destructive earthquakes with estimated magnitude about $M e=7$ have 
hit Central Italy (Di Mauro et al., 2008). Anyway, during the two decades 1989-2008, the area covered by the network has shown a low-moderate seismic activity and the epicentres of the few events with $M l \geq 5.0$ are located away enough from the network stations. Since the strongest effect occurs in the area near the epicentre, no significant variations in the local geomagnetic field is expected. During 2007 two light earthquakes $(M l \approx 4)$ occurred in proximity of AQU and CVT stations. In correspondence of these tectonic events, magnetic anomalies were observed in the total geomagnetic field.

\subsection{July 2007 event}

On 22 July at 17:26 UT an earthquake occurred $23 \mathrm{~km}$ away from CVT in NW direction (see Fig. 1). The earthquake magnitude was $M l=4$ and the hypocenter depth was $15.7 \mathrm{~km}$ (INGV Seismic Bulletin, 2007). In Fig. 3 the daily means of the total magnetic field differences for the couples of stations AQU-CVT, AQU-MDM and MDM-CVT in the period of time JD $=[170-260]$ are reported. For a better view of the differences trend a 13 days running mean is reported as well. The figure is divided into four intervals delimited by $\mathrm{JD}=170$, 194, 206, 226, 260. These intervals are chosen looking to the MDM-CVT curve. A linear fit of the differences is shown in each period of time as well. The black arrow and the vertical dashed-dotted line indicate the tectonic event occurred on 22 July (JD=203). The figure shows the presence of a magnetic anomaly in the second and in the third interval of time. This anomaly is certainly due to an increase of the CVT signal because the anomaly is present in the differences AQUCVT and MDM-CVT and is not evident in the differences AQU-MDM. Note that the trend of the AQU-MDM differences remains almost unchanged over the whole period of time. This trend is about $4 \cdot 10^{-3} \mathrm{nT} / \mathrm{day}$. The anomaly starts about a dozen of days before the earthquake, and ends about three weeks later with a total duration of about one month. The maximum amplitude of the anomaly was about $0.5 \mathrm{nT}$ three days after the tectonic event. Note that the differences MDM-CVT and AQU-CVT show the same trends both in the second interval of time (about $-4 \cdot 10^{-2} \mathrm{nT} /$ day) and in the third interval of time (about $2 \cdot 10^{-2} \mathrm{nT} /$ day). Obviously, the anomaly is more evident in the MDM-CVT differences than in the AQU-CVT differences because the MDM-CVT differentiated signal is less noisy. The ability to highlight magnetic anomalies using the differentiation procedure between two stations depends on the removal of the other magnetic changes. This removal is more efficient as the distance between the stations decreases (Davis and Johnston, 1983). In Fig. 1 can be noted that the distance between MDM and CVT is three times less than the distance between AQU and CVT. In Fig. 3 the geomagnetic activity indices $\Sigma K$, calculated by L'Aquila Observatory data, are reported as well. Figure 3 shows that there is no correlation between the magnetic anomaly and $\Sigma \mathrm{K}$.

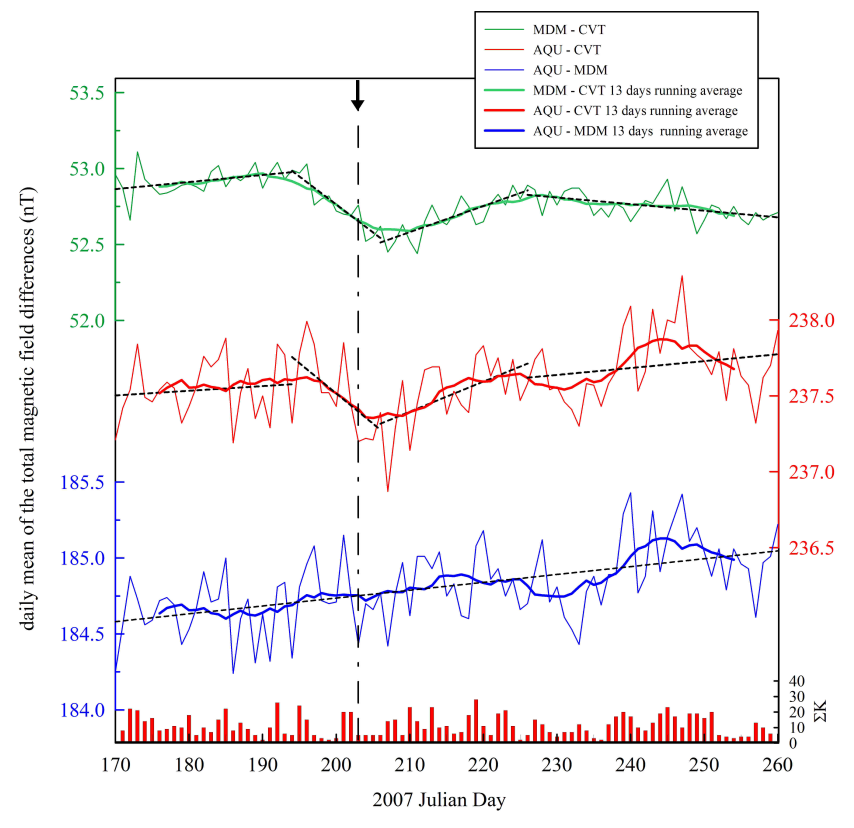

Fig. 3. The thin coloured lines represent the daily means of the total magnetic field differences for the couples of stations AQUCVT, AQU-MDM, MDM-CVT in the period of time JD $=[170$ 260] around the event of 22 July 2007 (black arrow). The thick coloured lines represent the 13 days running mean of the differences. The black dashed lines represent the linear fits of the differences in four period of time. The geomagnetic activity indices $\Sigma \mathrm{K}$ are reported as well.

\subsection{October 2007 event}

On 21 October at 03:55 UT an earthquake occurred $29 \mathrm{~km}$ away from AQU (see Fig. 1). The earthquake occurred only $18 \mathrm{~km}$ away from LEO, but unfortunately in that period the station was off. The earthquake magnitude was $M l=3.9$ and the hypocenter depth was $8.0 \mathrm{~km}$ (INGV Seismic Bulletin, 2007). In Fig. 4 are reported the daily means of the total magnetic field differences for the couples of stations AQU-CVT, AQU-MDM and MDM-CVT in the period of time $\mathrm{JD}=[250-340]$. For a better view of the differences trend a 13 days running mean is reported as well. The figure is divided into two intervals delimited by $\mathrm{JD}=250,294$, 320. The differences after JD=320 are not considered because of the occurrence of a magnetic storm. We want to stress that the storm is clearly evident in the total magnetic field of all the three stations. A linear fit of the differences is shown in each period of time as well. The black arrow and the vertical dashed-dotted line indicate the tectonic event of 21 October (JD=294). The figure shows the presence of a magnetic anomaly starting at the beginning of the second period of time. This anomaly is certainly due to a decrease of the AQU signal because the anomaly is present in the differences AQU-CVT and AQU-MDM, and is not evident in the differences MDM-CVT. Note that the 


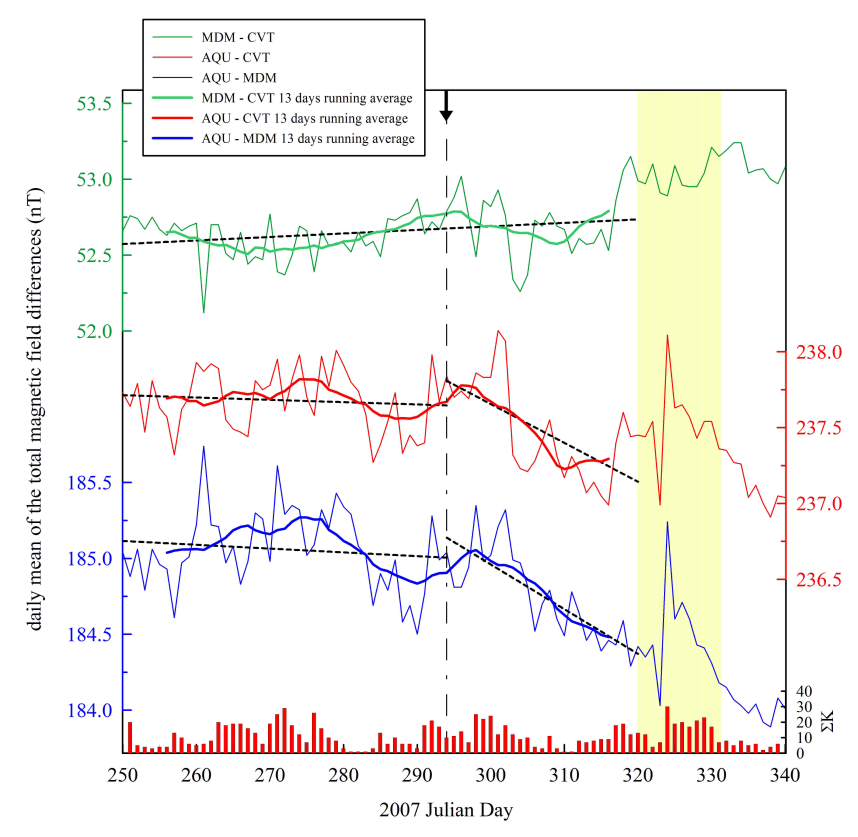

Fig. 4. As Fig. 3 for 21 October 2007 event. The yellow area underlines the influence of a geomagnetic storm occurred between $\mathrm{JD}=320$ and $\mathrm{JD}=331$.

trend of the MDM-CVT differences remains unchanged over the whole period of time. This trend can be quantified in about $3 \cdot 10^{-3} \mathrm{nT} /$ day. The anomaly starts about simultaneously with the tectonic event and remains evident till about the beginning of the magnetic storm $(\mathrm{JD} \approx 320)$ with a trend of about $-2.5 \cdot 10^{-2} \mathrm{nT} /$ day in both the differences AQU-CVT and AQU-MDM. In Fig. 4 the yellow area shows the temporal duration of the magnetic storm. The influence of magnetic storm is clearly evident in the AQU-CVT and AQU-MDM differences but it does not appear in the differences MDMCVT. This effect can be explained with the latitude dependence of the magnetic storm. Therefore, in the differences MDM-CVT the magnetic storm is not so evident because the latitudes of the two stations differ of only about $1^{\prime}$. Figure 4 shows that also in this event there is no correlation between the anomaly and the geomagnetic activity indices $\Sigma \mathrm{K}$.

\section{2006-2008 ULF analysis}

Electromagnetic emission possibly associated with tectonic activity can be observed in a wide range of frequencies (Hayakawa and Fujinawa, 1994; Johnston, 1997; Hayakawa and Molchanov, 2002). To mitigate earthquake hazard, some papers suggest that the study of ULF emissions is a good tool to investigate seismomagnetic effects as earthquake precursors (Hayakawa et at., 2007). The peculiarity of ULF waves lies in the capability to spread in the lithosphere from the hypocentral region up to the Earth's surface with a small attenuation over a long distance, whereas higher frequency sig- nals are subjected to a considerable attenuation. The ULF signal is a superposition of: 1) natural signal from solarterrestrial interaction (geomagnetic pulsation, etc.); 2) manmade noise; 3) natural signal from seismotectonic emissions. The signal associated with crustal activity is very weak so the problem is how to discriminate it from the others signals. In literature a numbers of methods of analysis to highlight these low signals have been developed (Hayakawa et al, 1996; Gotoh et al., 2004; Hattori et al., 2006) and some papers show the evidence of ULF signals due to large earthquakes at a distance greater than $100 \mathrm{~km}$ from the epicentre (Ohta et al., 2005; Hayakawa et al., 2007). In L'Aquila Observatory, in addition to the total geomagnetic field data, the $\mathrm{H}$ (NS), D (EW) and Z (vertical) components of the geomagnetic field are available by means of fluxgate sensors with $1 \mathrm{~Hz}$ sampling rate. Here we decided to apply the improved polarization analysis method proposed by Ida et al. (2008) to the complete 2006-2008 AQU dataset to isolate the ULF anomalous signals eventually present. Only the data of the local night time [00:00-04:00] UT are used to minimize the artificial noise and the effects of the geomagnetic pulsations. The polarization method is based on the calculation of the ratios $\mathrm{Z} / \mathrm{H}$ and $\mathrm{Z} / \mathrm{D}$ in the frequency domain. These ratios can increase in correspondence of seismogenic emissions. The geomagnetic field components are processed as follows. 1) The power spectral densities of each components $\mathrm{H}, \mathrm{D}, \mathrm{Z}$ are calculated daily in the interval of time corresponding to the local night time; the window function is Hanning, without overlapping, with a 1024 size. 2) The power spectral densities are selected around the frequency $0.01 \mathrm{~Hz}( \pm 0.003 \mathrm{~Hz})$. Hayakawa et al. (1996) have shown that this frequency is representative for ULF seismogenic emissions. 3) To make comparable the three components, they are normalized according to the following expression

$i_{n}=\left(A_{i}-\mu_{i}\right) / \sigma_{i}$

where $i$ represents the components $\mathrm{H}, \mathrm{D}$ and $\mathrm{Z}$ in the frequency domain; $A_{i}$ is the daily average of the $i$ component; $\mu_{i}$ and $\sigma_{i}$ are respectively the average value and the standard deviation of the $i$ component over the considered period of time. 4) Finally the normalized polarization ratios $\mathrm{Z}_{n} / \mathrm{H}_{n}$ and $Z_{n} / D_{n}$ are calculated. Figure 5 shows the results of the application of this method on the AQU dataset for the period 2006-2008. The grey arrow refers to the event of 21 October 2007 previously reported. Related to this event there is no anomalous increase in the polarization ratios before the earthquake as expected in the case of ULF emissions. The only increase in the ratios can be found after the earthquake but they are probably due to the magnetic storm previously described in Fig. 4. In any case, the analysis of the polarization ratios confirm the empirical law $0.025 R \leq M l-4.5$ ( $R$ is the epicentral distance, $M l$ is the earthquake local magnitude) for the detection of anomalous ULF signals before the earthquake occurrence (Hayakawa et al., 2007). This empirical law asserts that the threshold for ULF detection is an 

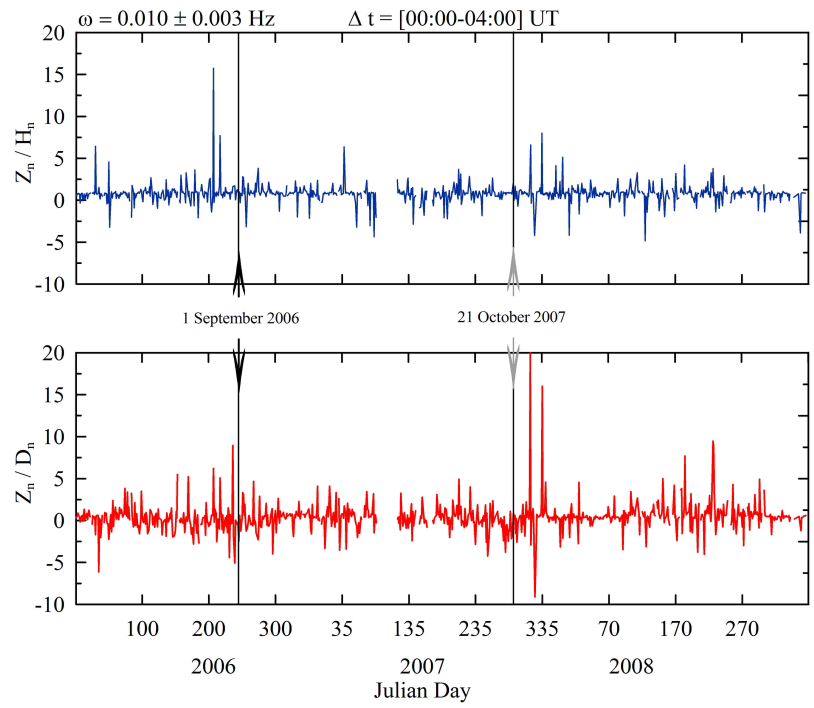

Fig. 5. Evolution of the normalized magnetic polarization ratios in the AQU station for the period of years 2006-2008. The black and grey arrows refer respectively to 1 September 2006 and to 21 October 2007 events. See text for details.

earthquake with magnitude $M l \geq 4$ occurred very close to the detection point. In Fig. 5 we report another tectonic event occurred in proximity of the AQU station. The black arrow refers to the earthquake occurred on 1 September 2006 at 15:12 UT $9 \mathrm{~km}$ away from AQU in NW direction. The earthquake magnitude was only $M l=3.1$, but the hypocenter was very shallow: it was only $1.8 \mathrm{~km}$ deep (INGV Seismic Bulletin, 2006). In Fig. 5 both the normalized polarization ratios $\mathrm{Z}_{n} / \mathrm{H}_{n}$ and $\mathrm{Z}_{n} / \mathrm{D}_{n}$ show a small increase before the earthquake. The ULF increase starts about three months before and concludes just after the earthquake. Figure 5 shows that both the polarization ratios have no remarkable variations in the months after the tectonic event and in the previous period of the ULF anomaly appearance. In any case this ULF anomaly is not clear enough to be sure that is certainly linked with the 1 September earthquake. To complete the analysis of this event, we show in Fig. 6 the daily means of the total magnetic field differences for the couple of stations AQU-CVT in the period JD $=[200-270] 2006$. Unfortunately in this period MDM station was off for technical problems (Masci et al., 2007), so we can show only the differences AQU-CVT. A 3 days running average of the differences is reported to better show the differences trend. The figure clearly shows the presence of a jump occurred approximately around JD=230 between two well defined levels. The amplitude of this jump is about $0.5 \mathrm{nT}$ during 4 days. The jump comes about two weeks before the earthquake occurred near the AQU station on 1 September 2006. In any case, since we can report only the AQU-CVT differences, we cannot single out if the anomaly is linked, as precursor signal, to the AQU data or otherwise to the CVT data.

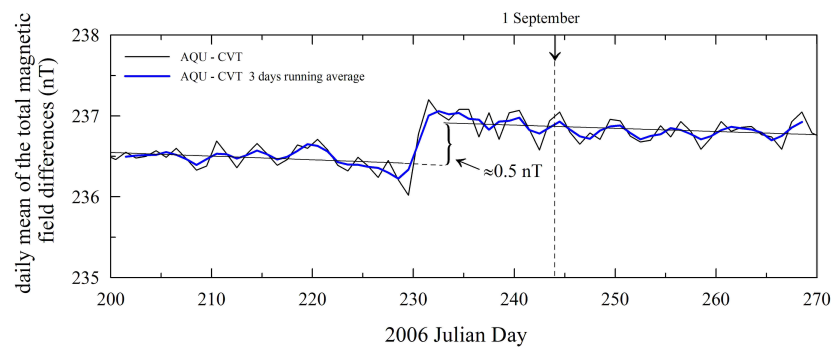

Fig. 6. Daily means of the total magnetic field differences for the couple of stations AQU-CVT in the period JD $=[200-270]$ in correspondence of the tectonic event of 1 September 2006 (black arrow). See text for details.

\section{Conclusions}

Two $M l \approx 4$ earthquakes occurred during 2007 in proximity of the AQU and CVT stations of the INGV tectonomagnetic network located in Central Italy. Magnetic anomalies that could be linked with these tectonic events were observed in the total geomagnetic field. The maximum amplitudes of the anomalies are about $0.5 \mathrm{nT}$. In the first case (22 July 2007 $M l=3.9$ earthquake near CVT station) the anomaly starts about two weeks before the earthquake and end about three weeks later. After this period the signal recover to the level preceding the anomaly. In the second case (21 October 2007 $M l=4$ earthquake near AQU Observatory) the anomaly starts about simultaneously with the earthquake and remains evident for about four weeks just before a magnetic storm appearance. In both the events there are no correlation with the geomagnetic activity indices $\Sigma \mathrm{K}$ obtained by L'Aquila Observatory data. To better investigate the second event, a ULF analysis, according to the improved polarization analysis method, has been carried out on the 2006-2008 AQU dataset. Unfortunately, we have not found an increase in the polarization ratios before the earthquake as expected. A small increase in the polarization ratios has been found before the $M l=3.1$ earthquake occurred only $9 \mathrm{~km}$ away from AQU on 1 September 2006. In any case, this ULF anomaly is not well defined to be sure that it is doubtless linked with the tectonic event. On the other hand, we have shown a magnetic anomaly in the AQU-CVT total magnetic field differences occurred two weeks before the earthquakes. Since the others stations of the network were off in this period, we are not able to distinguish if the anomaly is due, as earthquake precursory, to the AQU signal or otherwise to the CVT signal.

Acknowledgements. The authors thank their colleagues of L'Aquila Observatory for the basic support in the research activity. We also thank the two referees for their help in improving the paper.

Edited by: M. E. Contadakis

Reviewed by: P. F. Biagi and G. Vargemezis 


\section{References}

Bella, F., Biagi, P. F., Caputo, M., Della Monica, G., Ermini, A., Plastino, W., and Sgrigna, V.: Anomalies in different parameters related to the M=3.9 Gran Sasso earthquake (1992), Phys. Chem. Earth, 23, 959-963, 1998.

Biagi, P. F., Piccolo, R., Castellana, L., Ermini, A., Martellucci, S., Bellecci, C., Capozzi, V., Perna, G., Mochanov, O., and Hayakawa, M.: Variation in LF radio signal on the occasion of the recent seismic and volcanic activity in Southern Italy, Phys. Chem. Earth, 29, 551-557, 2004.

Biagi, P. F., Castellana, L., Maggipinto, T., Maggipinto, G., Minafra, A., Ermini, A., Capozzi, V., Perna, G., Solovieva, M., Rozhnoi, A., Molchanov, O. A., and Hayakawa, M.: Decrease in the electric intensity of VLF/LF radio signals and possible connections, Nat. Hazards Earth Syst. Sci., 7, 423-430, 2007, http://www.nat-hazards-earth-syst-sci.net/7/423/2007/.

Biagi, P. F., Castellana, L., Maggipinto, T., Loiacono, D., Augelli, V., Schiavulli, L., Ermini, A., Capozzi, V., Solovieva, M. S., Rozhnoi, A. A., Molchanov, O. A., and Hayakawa, M.: Disturbances in a VLF radio signal prior the $M=4.7$ offshore Anzio (central Italy) earthquake on 22 August 2005, Nat. Hazards Earth Syst. Sci., 8, 1041-1048, 2008,

http://www.nat-hazards-earth-syst-sci.net/8/1041/2008/.

Davis, P. M. and Jonhston, M. J. S.: Localized geomagnetic field changes near active faults in California 1974-1980, J. Geophys. Res., 88, 9452-9460, 1983.

Di Mauro, D., Di Persio, M., Lepidi, S., Masci, F., Mele, G., Meloni, A., and Palangio, P.: The INGV tectonomagnetic network in central Italy. Fifteen years of observation and future developments: an update, Ann. Geophys.-Italy, 51, 137-146, 2008.

Gotoh, K., Hayakawa, M., Smirnova, N. A., and Hattori, K.: Fractal analysis of seismogenic ULF emissions, Phys. Chem. Earth, 29, 419-424, 2004.

Hattori, K., Serita, A., Yoshino, C., Hayakawa, M., and Isezaki, N.: Singular spectral analysis and principal component analysis for signal discrimination of ULF geomagnetic data associated with 2000 Izu Island earthquake swarm, Phys. Chem. Earth, 31, 281291, 2006.

Hayakawa, M. and Fujinawa, Y. (Eds.): Electromagnetic Phenomena Related to Earthquake Prediction, Terra Scientific Publishing Co., Tokyo, 677 pp., 1994.

Hayakawa, M., Kawate, R., Molchanov, O. A., and Yumoto, K.: Results of ultra-low-frequency magnetic field measurements during the Guam earthquake of 8 August 1993, Geophys. Res. Lett., 23, 241-244, 1996.

Hayakawa, M. and Molchanov, O. A. (Eds.): Seismo Electomagnetics: Lithosphere-Atmosphere-Ionosphere Coupling, Terrapub, Tokio, 477 pp., 2002.

Hayakawa, M., Hattori, K., and Ohta, K.: Monitoring of ULF (ultralow-frequency) geomagnetic variations associated with earthquakes, Sensors, 7, 1108-1122, 2007.
Ida, Y., Yang, D., Li, Q., Sun, H., and Hayakawa, M.: Detection of ULF electromagnetic emissions as a precursor to an earthquake in China with an improved polarization analysis, Nat. Hazards Earth Syst. Sci., 8, 775-777, 2008, http://www.nat-hazards-earth-syst-sci.net/8/775/2008/.

INGV (Istituto Nazionale di Geofisica e Vulcanologia), Seismic Bulettin, Roma, 2006.

INGV (Istituto Nazionale di Geofisica e Vulcanologia), Seismic Bulettin, Roma, 2007.

Johnston, M. J. S. and Mueller, R. J.: Seismomagnetic observation during the 8 July 1986 magnitude 5.9 North Palm Springs earthquakes, Science, 237, 1201-1203, 1987.

Johnston, M. J. S.: Review of electrical and magnetic fields accompanying seismic and volcanic activity, Surv. Geophys., 18, 441-475, 1997.

Johnston, M. J. S. and Parrot, M.: Electromagnetic effects of earthquakes and volcanoes, Phys. Earth Planet. In., Special Volume, 105, 109-295, 1998.

Masci, F., Palangio, P., and Meloni, A.: The INGV tectonomagnetic network: 2004-2005 preliminary dataset analysis, Nat. Hazards Earth Syst. Sci., 6, 773-777, 2006,

http://www.nat-hazards-earth-syst-sci.net/6/773/2006/.

Masci, F., Palangio, P., Di Persio, M., and Di Lorenzo, C.: The development of the INGV tectonomagnetic network in the frame of the MEM Project, Nat. Hazards Earth Syst. Sci., 7, 473-478, 2007, http://www.nat-hazards-earth-syst-sci.net/7/473/2007/.

Masci, F., Palangio, P., and Di Persio, M.: The INGV tectonomagnetic network, Adv. Geosci., 14, 65-68, 2008, http://www.adv-geosci.net/14/65/2008/.

Mele, G., Meloni, A., and Palangio, P., A tectonomagnetic effect detected in Central Italy, Ann. Geofis., 37, 17-25, 1994.

Molchanov, O.: Social tension as precursor of large damaging earthquake: legend or reality?, Nat. Hazards Earth Syst. Sci., 8, 1259-1265, 2008, http://www.nat-hazards-earth-syst-sci.net/8/1259/2008/.

Molina, F., Armando E., Balia, R., Battelli, O., Bozzo, E., Budetta, E., Caneva, G., Ciminale, G., et al.: Geomagnetic survey of Italy at 1979.0. repeat Station Network and Magnetic Maps, Istituto Nazionale di Geofisica, Roma, 32 pp., 1994.

Mueller, R. J. and Johnston, M. J. S.: Review of magnetic field monitoring near active faults and volcanic calderas in California: 1974-1995, Phys. Earth Planet. In., Special Volume, 105, 131144, 1998.

Ohta, K., Watanabe, N., and Hayakawa, M.: The observation of ULF emissions at Nakatsugawa in possible association with the 2004 Mid Niigata Prefecture earthquake, Earth Planets Space, 57, 1103-1108, 2005.

Plastino, W. and Bella, F.: Radon groundwater monitoring at underground laboratories of Gran Sasso (Italy), Geophys. Res. Lett., 28, 2675-2677, 2001.

Stacey, F. D.: The seismomagnetic effect, Pure Appl. Geophys., 58, 5-22, 1964.

Tributsch, H.: When the snakes awake, Cambridge, MIT Press, 1983. 